\title{
Modal dynamics for positive operator measures
}

\author{
Jay Gambetta and H. M. Wiseman* \\ Centre for Quantum Dynamics, School of Science, Griffith University, Brisbane 4111, Australia
}

\begin{abstract}
The modal interpretation of quantum mechanics allows one to keep the standard classical definition of realism intact. That is, variables have a definite status for all time and a measurement only tells us which value it had. However, at present modal dynamics are only applicable to situations that are described in the orthodox theory by projective measures. In this paper we extend modal dynamics to include positive operator measures (POMs). That is, for example, rather than using a complete set of orthogonal projectors, we can use an overcomplete set of nonorthogonal projectors. We derive the conditions under which Bell's stochastic modal dynamics for projective measures reduce to deterministic dynamics, showing (incidentally) that Brown and Hiley's generalization of Bohmian mechanics [quant-ph/0005026, (2000)] cannot be thus derived. We then show how deterministic dynamics for positive operators can also be derived. As a simple case, we consider a Harmonic oscillator, and the overcomplete set of coherent state projectors (i.e. the Husimi POM). We show that the modal dynamics for this POM in the classical limit correspond to the classical dynamics, even for the nonclassical number state $|n\rangle$. This is in contrast to the Bohmian dynamics, which for energy eigenstates, the dynamics are always non-classical. .
\end{abstract}

\section{INTRODUCTION}

It has been almost eighty years since quantum theory emerged as a complete theory, yet fundamental debates still occur over its interpretation. These debates usually center around what has been called the measurement problem, which we argue is a twofold problem.

In quantum mechanics a complex vector $|\Psi(t)\rangle$, which belongs to a Hilbert space $\mathcal{H}$, is used to describe the state of a system, and its evolution is given by the Schrödinger equation. In the orthodox interpretation it is postulated that a system only has a definite value for an observable $\hat{A}$ (energy, position etc.) if the state $|\Psi(t)\rangle$ is an eigenstate, $\left|\Psi_{n}(t)\right\rangle$, of $\hat{A}$, in which case the value of $\hat{A}$ is the eigenvalue $a_{n}$ associated with $\left|\Psi_{n}(t)\right\rangle$. However, since the Schrödinger equations in linear it is possible for the system to be in a state which is a linear superposition of these eigenstates: $|\Psi(t)\rangle=\sum_{n} c_{n}(t)\left|\Psi_{n}(t)\right\rangle$. That is, the system can have two or more values of an observable at once or, we say, the value is not defined. To explain why only one of the values is obtained in a measurement we need to introduced an extra dynamical equation, the reduction equation. This equation for a measurement of observable $\hat{A}$ has the effect of collapsing the state, $|\Psi(t)\rangle \rightarrow\left|\Psi_{n}(t)\right\rangle$, thereby defining the value of the observable. Thus the standard view of reality (that variables have well defined values even when they are not observed) is lost. This is what we call the first problem of the measurement problem under the orthodox interpretation.

The second problem is concerned with choices: what observable is measured, and where the reduction occurs (the Heisenberg cut [1]). In the orthodox interpretation

\footnotetext{
*Electronic address: h.wiseman@griffith.edu.au
}

there is a classical world, which we live in, existing outside of the quantum world and allows us to define an apparatus which chooses the observable to be measured (the set of eigenstates to be collapsed into). The problem is, at what point do we place the distinction between system and apparatus? An apparatus is made of particles just like the system. Perhaps it is only an intelligent observer that collapses the wavefunction. But it is not clear how intelligence or consciousness can influence physics. Alternatively it can be argued that quantum mechanics should extend up the von Neumann chain [2] to include the entire universe, so $|\Psi(t)\rangle$ now labels the state of the universe. If this is the case then what is external to the universe which makes the quantum measurements?

In this paper we consider the modal interpretation of quantum mechanics $[3,4,5,6,7,8,9,10,11,12,13,14$, 15]. This interpretation's central goal is to keep the standard definition of reality intact, that observables have values even when not observed. In this interpretation to explain why only one value of $\hat{A}$ is actual for all time we introduce an extra state, the property state, $\left|\Psi_{n}(t)\right\rangle$. This state evolves stochastically with time (jumping between different values of $n$ ) and selects the present value of $\hat{A}, a_{n}$, from the set of possible values $\left\{a_{n}\right\}$. While $|\Psi(t)\rangle$ in effect chooses the weights for this stochastic jumping; that is, it acts as a guiding state. We define the unnormalized (signified by a tilde) property state as

$$
\left|\tilde{\Psi}_{n}(t)\right\rangle=\hat{\pi}_{n}(t)|\Psi(t)\rangle,
$$

where $\hat{\pi}_{n}(t)$ is a projector (acting in the total Hilbert space of the universe $\left.\mathcal{H}_{\text {uni }}\right)$ and satisfies $\sum_{n} \hat{\pi}_{n}(t)=\hat{1}$. The set of projectors, $\left\{\hat{\pi}_{n}(t)\right\}$ are labelled the preferred projective measure. The normalized property state is then defined as

$$
\left|\Psi_{n}(t)\right\rangle=\hat{\pi}_{n}(t)|\Psi(t)\rangle / \sqrt{N},
$$

where $N$ is a normalization constant. Note the set of 
property states depends on both the preferred projective measure and the guiding state. The guiding state $|\Psi(t)\rangle$ (as in the orthodox interpretation) is found from the Schrödinger equation

$$
d_{t}|\Psi(t)\rangle=-i \hat{H}_{\mathrm{uni}}(t)|\Psi(t)\rangle,
$$

where $\hat{H}_{\text {uni }}(t)$ is the Hamiltonian of the universe.

The reason why only one value for $\hat{A}$ is obtained upon a measurement of $\hat{A}$ at time $t$, is we choose the property states (i.e. choose $\left.\hat{\pi}_{n}(t)\right)$ so that $\hat{A}$ can be written as $\sum_{n} a_{n} \hat{\pi}_{n}(t)$. Now if $\left|\Psi_{n}(t)\right\rangle$ is the property state at time $t$, then $\hat{A}$ has the value $a_{n}$ at $t$ and if $\hat{A}$ is measured, this value is revealed. The probability that universe will be in the $n^{\text {th }}$ property state at time $t$ is given by

$$
P_{n}(t)=\left\langle\Psi(t)\left|\hat{\pi}_{n}(t)\right| \Psi(t)\right\rangle=\left\langle\tilde{\Psi}_{n}(t) \mid \tilde{\Psi}_{n}(t)\right\rangle,
$$

the standard Born probability. Thus the average of $\hat{A}$ over all possible property states will agree with $\langle\hat{A}\rangle$ found by the orthodox theory. However, unlike the orthodox interpretation this probability does not refer to the probability of observing results $a_{n}$ at time $t$ but to the probability of $A$ having value $a_{n}$ at time $t$.

For a given set of property states, there will be more than one observable, which can be assigned a definite value. Such and observable should, and from now on will, be referred to as a property or beable (after Bell $[3])$. Our notation for a property is

$$
Z=\left\{\left(z_{n}, \hat{\pi}_{n}(t)\right)\right\}
$$

where $z_{n}$ is the value of the property, which could be a real number, or a complex number, a statement (yes/no) or even a string of numbers. When the set $\left\{z_{n}\right\}$ are real (complex) numbers the property can be represented by a Hermitian (normal) operator

$$
\hat{Z}(t)=\sum_{n} z_{n} \hat{\pi}_{n}(t)
$$

Thus, in this interpretation, the first problem of the measurement problem is solved, at least for measurements of the preferred observable (property), as a measurement only tells us which value was possessed. For measurements of other observables see the discussion by Bohm for their interpretation [16]. However, the problem of choice still remains as we can choose a different preferred projective measure, $\left\{\hat{\pi}_{n}(t)\right\}$, which will give a different group of observables property status. This problem for modal interpretations has not been resolved in a wholly satisfactory way, and may never be.

The problem of choice is illustrated by the many variants of the modal interpretation. In the beable variant $[3,4,5,6,7]$ choice of the preferred projective measure is fundamental. That is, it is made independently of the guiding state. The problem is that many choices are viable [6]. In other modal theories $[8,9,10,11,12]$ the preferred projective measure depends on $|\Psi(t)\rangle$. These have been labelled by Bacciagaluppi and Dickson [9] as the atomic version of the modal interpretation. One assumes a preferred factorization of the universe $\mathcal{H}_{\alpha} \otimes \mathcal{H}_{\beta} \otimes \ldots \otimes \mathcal{H}_{\omega}$. (This seems to be necessary to avoid a Kochen-Specker type contradiction [17]). In this preferred factorization the spectral resolution of each subsystems $\rho_{\text {red }}^{\alpha}(t)=\sum_{n_{i}} w_{n_{i}} \hat{\pi}_{n_{i}}^{\alpha}(t)$ defines the preferred projective measure for the universe as

$$
\left\{\hat{\pi}_{n}(t)=\hat{\pi}_{n_{i}}^{\alpha}(t) \hat{\pi}_{n_{j}}^{\beta}(t) \ldots \hat{\pi}_{n_{z}}^{\omega}(t)\right\},
$$

where $n=\left(n_{i}, n_{j}, \ldots, n_{z}\right)$. This may seem like it has rectified the problem of choice but it still remains here in the choice of the preferred factorization. Another problem of choice can emerge in the atomic variant this being that for some guiding states there are many viable algorithms for defining the preferred projector. Note other variants of the modal interpretation $[6,13,15]$ which try to answer choice also encounter the above problems.

In this paper we do not propose to solve the problem of choice. To the contrary, we show that there is actually more choices to be made than what was previously realized. Specifically, it is not necessary to restrict the property to be of the form of Eq. (1.5), based upon orthogonal projectors. The extra choice is motivated by the fact that in the orthodox interpretation of quantum mechanics we can extend the theory of measurement from that using projective measures to that using positive operator measures (POMs). The former measurement (of observable Z) is described by a set of pairs as in Eq. (1.5), but here the $z_{n}^{\prime} s$ would be interpreted as measurement results. The latter measurement (of observable $\mathrm{Z}$ ) is described by a set of pairs

$$
Z=\left\{\left(z_{n}, \hat{F}_{n}(t)\right)\right\}
$$

where $\hat{F}_{n}(t)$ is referred to as a POM element [18] or effect [19]. These effects are positive and complete, with $\sum_{n} \hat{F}_{n}(t)=\hat{1}$. In this paper, we show that we can develop modal dynamics for preferred observables of the form in Eq. (1.8).

To agree with orthodox theory, our modal dynamics for POMs should generate the usual probability formula for POMs, which is

$$
P_{n}(t)=\left\langle\Psi(t)\left|\hat{F}_{n}(t)\right| \Psi(t)\right\rangle .
$$

Note that in general the number of possible results $\left(\sum_{n} 1\right)$ can be greater than the dimension of $\mathcal{H}_{\text {uni }}$. Some examples of POM measurements include informationally complete POMs $[20,21]$ and the Husimi or $Q$-function POM $[22,23]$. In the orthodox theory the reduction equation for POM-type measurement is $|\Psi\rangle \rightarrow \hat{M}_{n}|\Psi\rangle$ where $\hat{M}_{n}$ is referred to as the measurement operator and is defined such that $\hat{F}_{n}(t)=\hat{M}_{n}^{\dagger} \hat{M}_{n}[19]$.

Since the modal dynamics presented in Refs. $[3,4$, $5,7,8,9]$ assume that the property states are distinguishable, non-orthogonal states are not allowed in their analysis. In this paper we use Naimark's theorem [18] to 
extend the dynamics to include POMs. We begin in Sec. II by extending the stochastic dynamics introduced by Bell [3]. In Sec. III we consider the limits in which deterministic dynamics can be derived from the stochastic dynamics, for both the projective and the POM case. Along the way we show that Brown and Hiley's [25] generalization of Bohmian mechanics cannot be derived from Bell's modal dynamics. In Sec. IV we illustrate the POM case with an example of a universe consisting of only a single Harmonic oscillator. We show that when the Husimi POM (the overcomplete set of coherent state projectors), is used, the modal dynamics is consistent with classical mechanics in the classical (large excitation) limit. However when the modal dynamics is used to describe position property states (i.e. Bohmian mechanics) there is no recovery of classical behaviour in the classical limit. This illustrates the potential usefulness of modal dynamics of POMs.

\section{DYNAMICS FOR MODAL INTERPRETATIONS}

In this section we first briefly outline the modal dynamics for the projective case, which is basically a reproduction of the results presented in Refs. [3, 4, 5] and generalized in Refs. [7, 8, 9] to include time-dependent projectors. Secondly we use Naimark's theorem [18] to extend the dynamics to include positive operators.

\section{A. The Projective Case}

Before reproducing the standard results for the projector case we outline the general method used to describe a stochastic process, that jumps between $N$ distinct states. We define $P_{n}(t)$ as the probability that the system is in the $n^{t h}$ state at time $t$. Assuming a Markovian process, by which we mean that the probability of being in state $m$ at time $t+d t$ only depends on the state at time $t$, we can write the master equation as

$$
d_{t} P_{n}(t)=\sum_{m}\left[T_{n m}(t) P_{m}(t)-T_{m n} P_{n}(t)\right],
$$

where $T_{n m}$ is defined as

$$
T_{n m}(t)=\left[P_{n m}(t+d t, t)-P_{n m}(t, t)\right] / d t .
$$

Here $P_{n m}(t+d t, t)$ is a conditional probability and is read as the probability of the system being in state $n$ at time $t+d t$ given it was in state $m$ at time $t$. From this definition it follows that $\sum_{n} T_{n m}=0$.

For distinct states the conditional probability $P_{n m}(t, t)$ must be 0 for $n \neq m$. This allows us to interpret $T_{n m}(t) d t$ for $n \neq m$ as the transition probability, so we call $T_{n m}(t)$ the transition rates. For $n=m, P_{n n}(t, t)=1$ and $T_{n n}$ (which is negative) is a measure of the rate at which state $n$ losses probability.
Defining a probability current $J_{n m}(t)$ as

$$
J_{n m}(t)=T_{n m}(t) P_{m}(t)-T_{m n} P_{n}(t),
$$

results in $J_{n m}(t)=-J_{m n}(t)$ and allows us to rewrite the probability master equation as

$$
d_{t} P_{n}(t)=\sum_{m} J_{n m}(t) .
$$

Given $J_{n m}(t)$ and $P_{n}(t)$, there are many possible transition rates satisfying Eq. (2.4). One solution, chosen by Bell [3] is as follows.

For $J_{n m}(t)<0$,

$$
\begin{aligned}
& T_{n m}(t)=0, \\
& T_{m n}(t)=-J_{n m}(t) / P_{n}(t),
\end{aligned}
$$

and for $J_{n m}(t)>0$

$$
\begin{aligned}
& T_{n m}(t)=J_{n m}(t) / P_{m}(t), \\
& T_{m n}(t)=0 .
\end{aligned}
$$

This is only one of the infinitely many solutions. These are found by adding an extra term, $T_{n m}^{0}(t)$, to $T_{n m}(t)$, where $T_{n m}^{0}(t)$ is constrained only by

$$
T_{n m}^{0}(t) P_{m}(t)-T_{m n}^{0}(t) P_{n}(t)=0 .
$$

To make the link with quantum mechanics we say that the $N$ distinct states are the property states $\left\{\left|\Psi_{n}(t)\right\rangle\right\}$, and the set of properties which have definite values form the group $\mathcal{G}$, defined by elements

$$
\hat{Z}=\sum_{n} z_{n} \hat{\pi}_{n}(t)
$$

with the value of property $\hat{Z}$ being the corresponding $z_{n}$. The evolution of these values (jumping between $z_{n}$ ) is determined by the rates $T_{n m}(t)$, which themselves depend upon $J_{n m}(t)$.

By taking the time derivative of Eq. (1.4) we obtain the differential equation

$$
\begin{aligned}
d_{t} P_{n}(t)= & 2 \operatorname{Im}\left[\left\langle\Psi(t)\left|\hat{\pi}_{n}(t) \hat{H}_{\mathrm{uni}}(t)\right| \Psi(t)\right\rangle\right] \\
& +\left\langle\Psi(t)\left|d_{t}\left[\hat{\pi}_{n}(t)\right]\right| \Psi(t)\right\rangle,
\end{aligned}
$$

where we have used Eq. (1.3), the Schrödinger equation. This can be simplified by defining the Hermitian operator, $\hat{R}(t)$, for which

$$
d_{t} \hat{\pi}_{n}(t)=-i\left[\hat{R}(t), \hat{\pi}_{n}(t)\right]
$$

This allows us to rewrite Eq. (2.11) as

$$
d_{t} P_{n}(t)=2 \operatorname{Im}\left\{\left\langle\Psi(t)\left|\hat{\pi}_{n}(t)\left[\hat{H}_{\mathrm{uni}}(t)-\hat{R}(t)\right]\right| \Psi(t)\right\rangle\right\} .
$$

Comparing this with Eq. (2.4) and using the fact that $\sum_{m} \hat{\pi}_{m}(t)=\hat{1}$, one possible probability current (that was chosen by Bell [3]) is

$$
J_{n m}(t)=2 \operatorname{Im}\left\{\left\langle\Psi(t)\left|\hat{\pi}_{n}(t)\left[\hat{H}_{\mathrm{uni}}(t)-\hat{R}(t)\right] \hat{\pi}_{m}(t)\right| \Psi(t)\right\rangle\right\} .
$$


Note that this is only one of infinitely many possible currents, as we can add any current $J_{n m}^{0}(t)$ to $J_{n m}(t)$ which satisfies $\sum_{m} J_{n m}^{0}=0$, to give a valid probability current. For the purposes of this paper we only consider the simple solutions (not containing the extra $T_{n m}^{0}(t)$ and $J_{n m}^{0}(t)$ terms). For a discussion about these solution see [5] and [8].

\section{B. The Positive Operator Measure Case}

A typical example of a POM is the observable

$$
Z=\left\{\left(z_{n}, \frac{1}{B}\left|z_{n}\right\rangle\left\langle z_{n}\right|\right)\right\}
$$

where $\left\{\left|z_{n}\right\rangle\right\}$ forms an overcomplete basis in $\mathcal{H}_{\text {uni }}$, with $\sum_{n}\left|z_{n}\right\rangle\left\langle z_{n}\right|=\hat{1} B$. If the universe was in $\left|z_{n}\right\rangle$, then a measurement of this observable could with finite probability yield any of the values $\left\{z_{n}\right\}$. This clearly disagrees with the above description of modal dynamics. Thus we cannot treat non-orthogonal states in the same manner as orthogonal states.

POMs are not always generated from non-orthogonal states. Given any set of projectors $\left\{\hat{\Pi}_{n}(t)\right\}$ in a larger Hilbert space $\mathcal{K}=\mathcal{H}_{\text {uni }} \otimes \mathcal{H}_{\text {aux }}$ where $\mathcal{H}_{\text {aux }}$ is some auxiliary Hilbert space, it is well know that a set of POM elements can always be found by [18]

$$
\hat{F}_{n}(t)=\operatorname{Tr}_{\text {aux }}\left[\left(\hat{1}_{\text {uni }} \otimes \hat{\rho}_{\text {aux }}\right) \hat{\Pi}_{n}(t)\right]
$$

where $\hat{\rho}_{\text {aux }}$ is a state in $\mathcal{H}_{\text {aux }}$. For simplicity we define it as $\hat{\rho}_{\text {aux }}=|\phi\rangle\langle\phi|$. What is perhaps less well known is that given a POM $\left\{F_{n}(t)\right\}$ it is always possible to generate a projective measure $\left\{\hat{\Pi}_{n}(t)\right\}$ in a larger Hilbert space $\mathcal{H}_{\text {uni }} \otimes \mathcal{H}_{\text {aux }}$, where $\operatorname{dim}\left(\mathcal{H}_{\text {aux }}\right)$ is not necessarily equal to $\sum_{n} 1$. This is called Naimark's theorem $[18,24]$. It basically says that we can define a projector $\hat{\Pi}_{n}(t)$ such that

$$
\begin{aligned}
& \operatorname{Tr}_{\mathcal{H}_{\text {uni }}}\left[|\Psi(t)\rangle\langle\Psi(t)| \hat{F}_{n}(t)\right] \\
& =\operatorname{Tr}_{\mathcal{K}}\left[|\Psi(t)\rangle\langle\Psi(t)|\otimes| \phi\rangle\langle\phi| \hat{\Pi}_{n}(t)\right],
\end{aligned}
$$

for all $|\Psi(t)\rangle \in \mathcal{H}_{\text {uni }}$ and for $n=1, \ldots, N$ where $N$ is the number of POM elements in the POM. To work out the set $\left\{\hat{\Pi}_{n}(t)\right\}$ it is necessary to introduce another projector $\hat{\Pi}_{N+1}(t)$, such that

$$
\sum_{n}^{N+1} \hat{\Pi}_{n}(t)=\hat{1}_{\text {uni }+ \text { aux }}
$$

and

$$
\hat{\Pi}_{n}(t) \hat{\Pi}_{m}(t)=\hat{\Pi}_{n}(t) \delta_{n m}
$$

is satisfied for $n, m=1, \ldots, N+1$. The set of projectors in this enlarged Hilbert space is called the Naimark extension of $\hat{F}_{n}(t)$ [18]. A worked example of this is shown later in this section.
We now propose that to calculate modal dynamics for POMs, the preferred observable (determined by the preferred POM) defined by Eq. (1.8) becomes a property in $\mathcal{K}$. The set of properties which can have definite values is now defined by

$$
Z=\left\{\left(z_{n}, \hat{\Pi}_{n}(t)\right)\right\}
$$

and if $z_{n}$ is a number this is represented by the operator $\hat{Z}=\sum_{n}^{N+1} z_{n} \hat{\Pi}_{n}(t)$. The value $z_{N+1}$ is arbitrary. Note we expect that the preferred projective measure $\left\{\hat{\Pi}_{n}(t)\right\}$ may define more then one POM (set of POM elements) as preferred. The guiding state becomes

$$
|\Phi(t)\rangle=|\Psi(t)\rangle \otimes|\phi\rangle
$$

where $|\Psi(t)\rangle$ is still the solution to the Schrödinger equation (1.3). With this guiding state we can rewrite Eq. (1.9) as

$$
P_{n}(t)=\left\langle\Phi(t)\left|\hat{\Pi}_{n}(t)\right| \Phi(t)\right\rangle,
$$

which forces $P_{N+1}(t) \equiv 0$ for all time as the projector $\hat{\Pi}_{N+1}(t)$ projects into the null space of $|\Phi(t)\rangle$. The property states are defined in $\mathcal{K}$ as

$$
\left|\Phi_{n}(t)\right\rangle=\hat{\Pi}_{n}(t)|\Phi(t)\rangle / \sqrt{N^{\prime}},
$$

which now form an orthogonal (distinguishable) set and $N^{\prime}$ is the new normalization constant. Thus the standard analysis of modal dynamics now applies (Sec. II A). However, the property state in this extended Hilbert space is, in general, an entangled state (between the universe and the auxiliary system). This may raise interpretational difficulties, but we will leave this question for later work and just treat the above as a purely mathematical procedure to deal with POMs.

To work out the modal dynamics for POMs, as in the projector case, we need to find the probability current. Using Eq. (2.22) and Eq. (1.3), $J_{n m}(t)$ is

$$
\begin{aligned}
J_{n m}(t)= & 2 \operatorname{Im}\left\{\langle\Phi(t)| \hat{\Pi}_{n}(t)\left[\hat{H}_{\mathrm{uni}}(t) \otimes \hat{1}_{\mathrm{aux}}-\hat{R}^{\prime}(t)\right]\right. \\
& \left.\times \hat{\Pi}_{m}(t)|\Phi(t)\rangle\right\},
\end{aligned}
$$

where $\hat{R}^{\prime}(t)$ is Hermitian and defines the evolution of the projectors $\hat{\Pi}_{n}(t)$, by

$$
d_{t} \hat{\Pi}_{n}=-i\left[\hat{R}^{\prime}(t), \hat{\Pi}_{n}\right] .
$$

To illustrate the above we consider a simple example, a universe consisting of only a spin-1/2 system [18], and consider the observable $Z$ defined by the POM elements $\hat{F}_{n}=\frac{2}{3}\left|z_{n}\right\rangle\left\langle z_{n}\right|$, with $n$ being 1,2 , and 3 and $z_{n}=\exp (i 2 \pi n / 3)$. The states $\left|z_{n}\right\rangle$ are defined by

$$
\left|z_{n}\right\rangle=\frac{1}{\sqrt{2}}\left(z_{n}|1\rangle+z_{n}^{*}|2\rangle\right),
$$

where $|2\rangle$ and $|1\rangle$ are the eigenstates of $\hat{\sigma}_{z}$ (the Pauli spin matrix). In the Bloch sphere these states all lay in the $x-y$ plane with an angular separation of $2 \pi / 3$. 
Using Naimark's theorem we extend this 2-dimensional Hilbert space to a 4-dimensional Hilbert space, where it can be shown (using Eqs. (2.18) and (2.19)) that the four projectors are [18]

$$
\begin{aligned}
& \hat{\Pi}_{1}=\hat{F}_{1} \otimes|\phi\rangle\left\langle\phi|-\sqrt{2} / 3| z_{1}\right\rangle\left\langle z_{2}|\otimes| \phi\right\rangle\left\langle\phi^{\prime}|-\sqrt{2} / 3| z_{2}\right\rangle\left\langle z_{1}|\otimes| \phi^{\prime}\right\rangle\left\langle\phi|+1 / 3| z_{2}\right\rangle\left\langle z_{2}|\otimes| \phi^{\prime}\right\rangle\left\langle\phi^{\prime}\right|, \\
& \hat{\Pi}_{2}=\hat{F}_{2} \otimes|\phi\rangle\left\langle\phi|+\sqrt{2} / 3| z_{2}\right\rangle\left\langle z_{2}|\otimes| \phi\right\rangle\left\langle\phi^{\prime}|+\sqrt{2} / 3| z_{2}\right\rangle\left\langle z_{2}|\otimes| \phi^{\prime}\right\rangle\left\langle\phi|+1 / 3| z_{2}\right\rangle\left\langle z_{2}|\otimes| \phi^{\prime}\right\rangle\left\langle\phi^{\prime}\right|, \\
& \hat{\Pi}_{3}=\hat{F}_{3} \otimes|\phi\rangle\left\langle\phi|+\sqrt{2} / 3| z_{3}\right\rangle\left\langle z_{2}|\otimes| \phi\right\rangle\left\langle\phi^{\prime}|+\sqrt{2} / 3| z_{2}\right\rangle\left\langle z_{3}|\otimes| \phi^{\prime}\right\rangle\left\langle\phi|+1 / 3| z_{2}\right\rangle\left\langle z_{2}|\otimes| \phi^{\prime}\right\rangle\left\langle\phi^{\prime}\right|, \\
& \hat{\Pi}_{4}=\hat{1} \otimes\left|\phi^{\prime}\right\rangle\left\langle\phi^{\prime}|-| z_{2}\right\rangle\left\langle z_{2}|\otimes| \phi^{\prime}\right\rangle\left\langle\phi^{\prime}\right| .
\end{aligned}
$$

The two states $|\phi\rangle$ and $\left|\phi^{\prime}\right\rangle$ form a set of orthogonal basis states of the auxiliary Hilbert space and $\hat{\Pi}_{4}$ is an added projector needed to complete the set of projectors.

The modal dynamics for these states is formulated as follows. By Eq. (2.21) and Eq. (2.23) the three possible property states are $\left\{\left|\Phi_{n}(t)\right\rangle=\hat{\Pi}_{n}\left|\phi_{1}\right\rangle|\Psi(t)\rangle / \sqrt{N^{\prime}}\right\}$, where $n=1,2,3$. The fourth projector is not included as for all possible states it projects into the null space of $|\Phi(t)\rangle$. The observable $Z=\left\{\left(z_{n}, \hat{F}_{n}\right)\right\}$ becomes the property $Z=\left\{\left(z_{n}, \hat{\Pi}_{n}\right)\right\}$ (or $\hat{Z}=\sum_{n} z_{n} \hat{\Pi}_{n}$ since for all $n, z_{n}$ is a number), and for this example the possible values are; $z_{1}=e^{i 2 \pi / 3}, z_{2}=e^{i 4 \pi / 3}, z_{3}=1$. The stochastic evolution between these values is then found from the transition rates $T_{n m}(t)$, which in turn require a specification of $J_{n m}(t)$. For simplicity, and to illustrate the significance of enlarging the Hilbert space, we assume that there is no evolution, that is $J_{n m}(t)=T_{n m}(t)=0$. Thus once we first assign a property state (based on the initial probability distribution) it remains in this property state for all time. Now we assume that the initial state of the universe is in one of the three non-orthogonal states, say $|\Psi(0)\rangle=\left|z_{1}\right\rangle$. Unlike in the projector (orthogonal) case we expect there to be some probability for the universe to be in any of the 3 possible property states $\left|\Phi_{n}(t)\right\rangle$. This does occur as in general $\hat{\Pi}_{n}|\phi\rangle\left|z_{1}\right\rangle$ is non-zero for all $n$.

\section{CONTINUOUS MODAL DYNAMICS}

In this section we investigate the continuum limit of modal dynamics. This has been previously done by Sudbery and Vink in Refs. [4] and [5] respectively, where it was shown that Bohmian mechanics can be obtained by choosing the appropriate preferred projective measure (property states). In this section we briefly outline their work, then extend it to show that the Brown and Hiley generalization of Bohmian mechanics to include the momentum representations [25] is not the continuum limit of Bell's modal dynamics. We also present an alternative (we believe easier) method for finding the continuous trajectories, that works when the modal dynamics has a continuum limit.

\section{A. Bohmian Mechanics}

In Bohmian mechanics [16] the preferred projective measure is the one associated with the position basis. That is, the property is the position of the system $\left\{X_{j}\right\}$ (vector notation) and the wavefunction $\Psi\left(\left\{x_{j}\right\}, t\right)=$ $\left\langle\left\{x_{j}\right\} \mid \Psi(t)\right\rangle$ is then interpreted as a field which guides the position in a non-classical way. In Bohm's original papers he showed that this non-classical behavior could be represent by an extra potential in the HamiltonianJacobi equation, the quantum potential which depends on $\Psi\left(\left\{x_{j}\right\}, t\right)$.

In this paper we do not introduce the quantum potential, but instead describe Bohm trajectories with reference to a continuous probability current $J_{k}\left(\left\{x_{j}\right\}, t\right)$. We also consider $|\Psi(t)\rangle$ to belong to a tensor product $\mathcal{H}_{x} \otimes \mathcal{H}^{\prime}$, where $\mathcal{H}_{x}$ is the Hilbert space containing the position eigenstates $\left|\left\{x_{j}\right\}\right\rangle$. Then the wavefunction becomes a vector given by,

$$
\left|\tilde{\psi}\left(\left\{x_{j}\right\}, t\right)\right\rangle=\left\langle\left\{x_{j}\right\} \mid \Psi(t)\right\rangle \in \mathcal{H}^{\prime} .
$$

This allows us to take into account a Hilbert space for the universe which is larger than that for the position of the system. Here we see that $\left|\left\{x_{j}\right\}\right\rangle\left|\tilde{\psi}\left(\left\{x_{j}\right\}, t\right)\right\rangle$ is the continuous equivalent of our unnormalized property state, and the properties are the position operators $\left\{\hat{X}_{k}\right\}$. With this property state we can define a probability density as

$$
P\left(\left\{x_{j}\right\}, t\right)=\left\langle\tilde{\psi}\left(\left\{x_{j}\right\}, t\right) \mid \tilde{\psi}\left(\left\{x_{j}\right\}, t\right)\right\rangle
$$

which obeys the continuity equation

$$
\partial_{t} P\left(\left\{x_{j}\right\}, t\right)=-\sum_{k} \partial_{x_{k}} J_{k}\left(\left\{x_{j}\right\}, t\right),
$$

where $J_{k}\left(\left\{x_{j}\right\}, t\right)$ depends on the form of $H_{\text {uni }}(t)$. As in the modal case there is not a unique solution to $J_{k}\left(\left\{x_{j}\right\}, t\right)$ as for example in three dimensions we can add any vector field $\nabla \times \mathbf{A}\left(\left\{x_{j}\right\}, t\right)$ to $\mathbf{J}\left(\left\{x_{j}\right\}, t\right)$ as $\nabla \cdot \nabla \times \mathbf{A}\left(\left\{x_{j}\right\}, t\right) \equiv 0$.

We define a velocity field $v_{k}\left(\left\{x_{j}\right\}, t\right)$ implicitly by

$$
J_{k}\left(\left\{x_{j}\right\}, t\right)=v_{k}\left(\left\{x_{j}\right\}, t\right) P\left(\left\{x_{j}\right\}, t\right) .
$$

Bohmian trajectories for $\left\{x_{j}\right\}(t)$ are then defined by

$$
d_{t} x_{k}(t)=\left.v_{k}\left(\left\{x_{j}\right\}, t\right)\right|_{x_{k}=x_{k}(t)} .
$$


Probability enters only through the initial conditions, $\left\{x_{k}(0)\right\}$. This is only one of the possible continuous trajectories which satisfy Eq. (3.2). Other possibilities include stochastic approaches; see Ref. [5] and references within. It should be noted that so far nothing has specified that $\left\{x_{j}\right\}$ must be positions, and in fact Brown and Hiley [25] develop a formalism where $\left\{x_{j}\right\}$ can be either position or momentum. As examples, they consider a simple universe (a single 1-D particle) and derive $d_{t} x(t)$ and $d_{t} p(t)$ for a linear, quadratic and cubic potential.

\section{Bohmian mechanics as the continuous limit of discrete modal dynamics}

To demonstrate that the modal dynamics does give Bohmian mechanics as its continuum limit, first consider the Hamiltonian

$$
\begin{aligned}
\hat{H}_{\mathrm{uni}}(t)= & \hat{A}(t)+\sum_{k} \hat{B}_{k}(t) \hat{Y}_{k}+\sum_{k} \hat{Y}_{k} \hat{B}_{k}^{\dagger}(t) \\
& +\sum_{k} \hat{C}_{k}(t) \hat{Y}_{k}^{2}+\sum_{k} \hat{Y}_{k}^{2} \hat{C}_{k}^{\dagger}(t),
\end{aligned}
$$

where $\hat{A}(t), \hat{B}_{k}(t)$ and $\hat{C}_{k}(t)$ are arbitrary functions of the operators $\left\{\hat{X}_{k}\right\}$ and the rest of the universe, and $\left\{\hat{Y}_{k}\right\}$ are the conjugate operators to $\left\{\hat{X}_{k}\right\}$. That is, $\left[\hat{Y}_{j}, \hat{X}_{k}\right]=$ $-i \delta_{j k}$.

To calculate $d_{t} x_{k}(t)$ in Eq. (3.5) we need to calculate the velocity field $v_{k}\left(\left\{x_{j}\right\}, t\right)$, which in turn requires calculation of $J_{k}\left(\left\{x_{j}\right\}, t\right)$. Taking the derivative of Eq. (3.2) and using the Schrödinger equation (Eq. (1.3)) we can write

$$
\begin{aligned}
d_{t} P\left(\left\{x_{j}\right\}, t\right)= & 2 \operatorname{Im}\left[\left\langle\Psi(t) \mid\left\{x_{j}\right\}\right\rangle\left\langle\left\{x_{j}\right\}\left|\hat{H}_{\text {uni }}(t)\right| \Psi(t)\right\rangle\right] \\
= & -2 \sum_{k} \operatorname{Re}\left[\left\langle\tilde{\psi}\left(\left\{x_{j}\right\}, t\right)\left|\left\{2 \operatorname{Re}\left[\hat{B}_{k}\left(\left\{x_{j}\right\}, t\right)\right] \partial_{x_{k}}+\partial_{x_{k}} \operatorname{Re}\left[\hat{B}_{k}\left(\left\{x_{j}\right\}, t\right)\right]\right\}\right| \tilde{\psi}\left(\left\{x_{j}\right\}, t\right)\right\rangle\right] \\
& -2 \sum_{k} \operatorname{Im}\left[\left\langle\tilde{\psi}\left(\left\{x_{j}\right\}, t\right)\left|\left\{2 \operatorname{Re}\left[\hat{C}_{k}\left(\left\{x_{j}\right\}, t\right)\right] \partial_{x_{k}}^{2}+2 \partial_{x_{k}} \hat{C}_{k}^{\dagger}\left(\left\{x_{j}\right\}, t\right) \partial_{x_{k}}+\partial_{x_{k}}^{2} \hat{C}_{k}^{\dagger}\left(\left\{x_{j}\right\}, t\right)\right\}\right| \tilde{\psi}\left(\left\{x_{j}\right\}, t\right)\right\rangle\right],
\end{aligned}
$$

which can be shown to be

$$
d_{t} P\left(\left\{x_{j}\right\}, t\right)=-\sum_{k} \partial_{x_{k}}\left\{\operatorname{Re}\left[\left\langle\tilde{\psi}\left(\left\{x_{j}\right\}, t\right)\left|\left\{2 \operatorname{Re}\left[\hat{B}_{k}\left(\left\{x_{j}\right\}, t\right)\right]-4 i \operatorname{Re}\left[\hat{C}_{k}\left(\left\{x_{j}\right\}, t\right)\right] \partial_{x_{k}}-2 i \partial_{x_{k}}\left[\hat{C}_{k}^{\dagger}\left(\left\{x_{j}\right\}, t\right)\right]\right\}\right| \tilde{\psi}\left(\left\{x_{j}\right\}, t\right)\right\rangle\right]\right\}
$$

Comparing this with Eq. (3.3) gives

$$
J_{k}\left(\left\{x_{j}\right\}, t\right)=\operatorname{Re}\left[\left\langle\tilde{\psi}\left(\left\{x_{j}\right\}, t\right)\left|\left\{2 \operatorname{Re}\left[\hat{B}_{k}\left(\left\{x_{j}\right\}, t\right)\right]-4 i \operatorname{Re}\left[\hat{C}_{k}\left(\left\{x_{j}\right\}, t\right)\right] \partial_{x_{k}}-2 i \partial_{x_{k}}\left[\hat{C}_{k}^{\dagger}\left(\left\{x_{j}\right\}, t\right)\right]\right\}\right| \tilde{\psi}\left(\left\{x_{j}\right\}, t\right)\right\rangle\right]
$$

For simplicity we consider the case when $\hat{A}(t)=\hat{V}(\hat{X})$, $\hat{B}_{k}(t)=0$, and $\hat{C}(t)=\hat{C}^{\dagger}(t)=\hat{1} / 4 M$, describing for example an electron in a 1-D potential, with the operator nature of $\hat{1}$ and $\hat{V}$ signifying operation on the Hilbert space for the internal structure of the electron. For this example $J(x, t)$ becomes

$$
J(x, t)=\frac{1}{M} \operatorname{Im}\left[\left\langle\tilde{\psi}(x, t)\left|\partial_{x}\right| \tilde{\psi}(x, t)\right\rangle\right],
$$

and thus

$$
d_{t} x(t)=\left.\frac{1}{M} \operatorname{Im}\left[\frac{\left\langle\tilde{\psi}(x, t)\left|\partial_{x_{k}}\right| \tilde{\psi}(x, t)\right\rangle}{\langle\tilde{\psi}(x, t) \mid \tilde{\psi}(x, t)\rangle}\right]\right|_{x=x(t)} .
$$

To simplify this we can rewrite $|\tilde{\psi}(x, t)\rangle$ as

$$
|\tilde{\psi}(x, t)\rangle=\sum_{j} R_{j}(x, t) \exp \left[i S_{j}(x, t)\right]|j\rangle
$$

where $R_{j}(x, t)$ and $S_{j}(x, t)$ are real functions and $\{|j\rangle\}$ is an orthonormal basis set, which for example spans the Hilbert space of the internal structure of the electron. Then it simplifies to

$$
d_{t} x(t)=\left.\frac{\sum_{j} R_{j}^{2}(x, t) \partial_{x}\left[S_{j}(x, t)\right]}{M \sum_{j} R_{j}^{2}(x, t)}\right|_{x=x(t)}
$$

To compare this to the modal dynamics defined in Sec. II we have to discretize $X$. In [5] and [4] this is done by defining a lattice of size $N$ and lattice separation $\epsilon$. Thus the values of the property $X$ denoted $x$ become $x_{n}=\epsilon n$, and the preferred projective measure becomes $\left\{\hat{\pi}_{n}=|\epsilon n\rangle\langle\epsilon n| \otimes \hat{1}\right\}$. With this preferred projective measure the property state Eq. (1.2) becomes $\left|\tilde{\Psi}_{n}(t)\right\rangle=|\epsilon n\rangle\langle\epsilon n \mid \Psi(t)\rangle=|\epsilon n\rangle\left|\tilde{\psi}_{n}(t)\right\rangle$ where $\left|\tilde{\psi}_{n}(t)\right\rangle$ is an unnormalized state existing in $\mathcal{H}^{\prime}$. Using the results of 
Sec. II A the probability current is

$$
J_{n m}(t)=2 \operatorname{Im}\left[\left\langle\tilde{\psi}_{n}(t)\left|\left\langle\epsilon n\left|\hat{H}_{\mathrm{uni}}\right| \epsilon m\right\rangle\right| \tilde{\psi}_{m}(t)\right\rangle\right],
$$

and the discretized version of the Hamiltonian is

$$
\begin{aligned}
\left\langle\epsilon n\left|\hat{H}_{\mathrm{uni}}\right| \epsilon m\right\rangle= & -\left(\delta_{n, m+1}+\delta_{n, m-1}-2 \delta_{n, m}\right) / 2 M \epsilon^{2} \\
& +\hat{V}(\epsilon n) \delta_{n, m} .
\end{aligned}
$$

This gives

$$
\begin{aligned}
J_{n m}(t)= & \frac{-1}{M \epsilon^{2}} \operatorname{Im}\left[\left\langle\tilde{\psi}_{n}(t) \mid \tilde{\psi}_{n-1}(t)\right\rangle \delta_{n, m+1}\right. \\
& \left.+\left\langle\tilde{\psi}_{n}(t) \mid \tilde{\psi}_{n+1}(t)\right\rangle \delta_{n, m-1}\right]
\end{aligned}
$$

Taylor expanding $\left|\tilde{\psi}_{n+1}(t)\right\rangle$ and $\left|\tilde{\psi}_{n-1}(t)\right\rangle$ gives

$$
\begin{aligned}
J_{n m}(t)= & \frac{1}{M \epsilon} \operatorname{Im}\left\{\left\langle\tilde{\psi}_{n}(t)\right| \Delta_{\epsilon}\left[\left|\tilde{\psi}_{n}(t)\right\rangle\right] \delta_{n, m+1}\right. \\
& \left.-\left\langle\psi_{n}(t)\right| \Delta_{\epsilon}\left[\left|\tilde{\psi}_{n}(t)\right\rangle\right] \delta_{n, m-1}+O(\epsilon)\right\},
\end{aligned}
$$

where $\Delta_{\epsilon}$ is the discretized version of a derivative. As in the continuous case we write $\left|\tilde{\psi}_{n}(t)\right\rangle$ in terms of the real functions $S_{j}(\epsilon n, t)$ and $R_{j}(\epsilon n, t)$ which results in $J_{n m}(t)$ becoming

$$
\begin{aligned}
J_{n m}(t)= & \frac{1}{M \epsilon}\left\{\sum_{j} R_{j}^{2}(\epsilon n) \Delta_{\epsilon}\left[S_{j}(\epsilon n)\right] \delta_{n, m+1}\right. \\
& \left.-\sum_{j} R_{j}^{2}(\epsilon n) \Delta_{\epsilon}\left[S_{j}(\epsilon n)\right] \delta_{n, m-1}+O(\epsilon)\right\} .
\end{aligned}
$$

Since $1 / \epsilon \gg 1$, in the $\epsilon \rightarrow 0$ limit (continuum limit) we can neglect the higher order terms in the above expression for $J_{n m}(t)$. That is the only terms which will contribute are the transitions from $m$ to $m-1$ or $m$ to $m+1$. Because of this we can write

$$
\begin{aligned}
& J_{n(n+1)}(t)=-\frac{1}{M \epsilon} \sum_{j} R_{j}^{2}(\epsilon n) \Delta_{\epsilon}\left[S_{j}(\epsilon n)\right] \\
& J_{n(n-1)}(t)=\frac{1}{M \epsilon} \sum_{j} R_{j}^{2}(\epsilon n) \Delta_{\epsilon}\left[S_{j}(\epsilon n)\right] .
\end{aligned}
$$

If $\sum_{j} R_{j}^{2} \Delta_{\epsilon}\left[S_{j}(\epsilon n)\right]>0$ (implies $J_{n(n+1)}(t)<0$ and $\left.J_{n(n-1)}(t)>0\right)$ then by Eqs. $(2.5)-(2.8)$,

$$
\begin{aligned}
& T_{(n+1) n}(t)=\frac{\sum_{j} R_{j}^{2}(\epsilon n) \Delta_{\epsilon}\left[S_{j}(\epsilon n)\right]}{M \epsilon \sum_{j} R_{j}^{2}(\epsilon n)} \\
& T_{(n-1) n}(t)=0 .
\end{aligned}
$$

If $\sum_{j} R_{j}^{2} \Delta_{\epsilon}\left[S_{j}(\epsilon n)\right]<0$ (implies $J_{n(n+1)}(t)>0$ and $\left.J_{n(n-1)}(t)<0\right)$ then by Eqs. $(2.5)-(2.8)$

$$
\begin{aligned}
& T_{(n+1) n}(t)=0 \\
& T_{(n-1) n}(t)=-\frac{\sum_{j} R_{j}^{2}(\epsilon n) \Delta_{\epsilon}\left[S_{j}(\epsilon n)\right]}{M \epsilon \sum_{j} R_{j}^{2}(\epsilon n)} .
\end{aligned}
$$

These transition rates imply that in an interval $d t$ the average displacement $d x$ will be

$$
\begin{aligned}
E[d x] & =\epsilon T_{(n+1) n} d t-\epsilon T_{(n-1) n} d t \\
& =\frac{\sum_{j} R_{j}^{2}(\epsilon n) \Delta_{\epsilon}\left[S_{j}(\epsilon n)\right]}{M \sum_{j} R_{j}^{2}(\epsilon n)} d t+O(\epsilon) d t
\end{aligned}
$$

Provided $S_{j}(\epsilon n)$ and $R_{j}(\epsilon n)$ are continuous, the average $E[d x]$ reduces to Eq. (3.13) as $\epsilon \rightarrow 0$. However, to show that the trajectories are smooth and deterministic from the initial $\left\{x_{k}(0)\right\}$ we also require that the dispersion $E\left[d x^{2}\right]$ goes to zero in the continuum limit. This is the case since

$$
E\left[d x^{2}\right]=\epsilon^{2} T_{(n+1) n}+\epsilon^{2} d t T_{(n-1) n} d t=O(\epsilon) d t
$$

which goes to zero as $\epsilon \rightarrow 0$.

\section{Hamiltonians for which Bohmian mechanics is not the continuous limit of discrete modal dynamics}

The above demonstrates that in the continuum limit modal dynamics becomes Bohmian Mechanics. However, if we consider a Hamiltonian of the form

$$
H_{\mathrm{uni}}=\kappa \hat{Y}^{3}+V(\hat{X})
$$

we find that the continuous limit of discrete (Bell-type) modal dynamics is not Bohmian mechanics. Note this Hamiltonian is unreasonable if $\hat{X}$ is position as this says we have a cubic dependence on momentum, which is not present in any natural Hamiltonians. However, if $\hat{X}$ corresponds to momentum and $-\hat{Y}$ to position, (which occurs in in Brown and Hiley's [25] extension of Bohmian mechanics to include the momentum representation), then this Hamiltonian is valid.

As before if we discretize the value of $X, x \rightarrow x_{n}=\epsilon n$, the probability current again will be given by Eq. (3.14). However, the discretized version of the Hamiltonian in this case is

$$
\begin{aligned}
\left\langle\epsilon n\left|\hat{H}_{\mathrm{uni}}\right| \epsilon m\right\rangle= & \frac{\kappa}{\epsilon^{3}}\left(i \delta_{n, m+3}-i \delta_{n, m-3}-3 i \delta_{n, m+1}\right. \\
& \left.+3 i \delta_{n, m-1}\right)+V(\epsilon n) \delta_{n, m}
\end{aligned}
$$

which results in 


$$
\begin{aligned}
J_{n m}(t)= & \frac{\kappa}{\epsilon^{3}} \operatorname{Im}\left[i \delta_{n, m+3}\left\langle\tilde{\psi}_{n}(t) \mid \tilde{\psi}_{n-3}(t)\right\rangle-i \delta_{n, m-3}\left\langle\tilde{\psi}_{n}(t) \mid \tilde{\psi}_{n+3}(t)\right\rangle-3 i \delta_{n, m+1}\left\langle\tilde{\psi}_{n}(t) \mid \tilde{\psi}_{n-1}(t)\right\rangle\right. \\
& \left.+3 i \delta_{n, m-1}\left\langle\tilde{\psi}_{n}(t) \mid \tilde{\psi}_{n+1}(t)\right\rangle\right] .
\end{aligned}
$$

Taylor expanding this gives a rather large expression, but since $\left\langle\tilde{\psi}_{n}(t) \mid \tilde{\psi}_{n}(t)\right\rangle$ is a real number and $1 / \epsilon^{3} \gg 1 / \epsilon^{2}$ in the $\epsilon \rightarrow 0$ limit we can ignore all orders of the Taylor expansion. This allows us to write

$$
\begin{aligned}
J_{n m}(t)= & \frac{\kappa\left\langle\tilde{\psi}_{n}(t) \mid \tilde{\psi}_{n}(t)\right\rangle}{\epsilon^{3}}\left[\delta_{n, m+3}-\delta_{n, m-3}-3 \delta_{n, m+1}\right. \\
& \left.+3 \delta_{n, m-1}\right] .
\end{aligned}
$$

Since $\left\langle\tilde{\psi}_{n}(t) \mid \tilde{\psi}_{n}(t)\right\rangle$ is always positive, for $\kappa>0$ the transitions rates defined in Eqs. (2.5) - (2.8) become

$$
\begin{aligned}
& T_{(n-3) n}(t)=0 \\
& T_{(n+3) n}(t)=\frac{\kappa}{\epsilon^{3}} \\
& T_{(n-1) n}(t)=\frac{3 \kappa}{\epsilon^{3}} \\
& T_{(n+1) n}(t)=0 .
\end{aligned}
$$

These transition rates imply that in an interval $d t$ the average displacement $d x$ will be

$$
M[d x]=3 \epsilon T_{(n+3) n} d t-\epsilon T_{(n-1) n} d t=0
$$

and the dispersion will be

$$
M\left[d x^{2}\right]=9 \epsilon^{2} T_{(n+1) n}+\epsilon^{2} d t T_{(n-1) n} d t=12 \kappa d t / \epsilon
$$

which diverges as $\epsilon \rightarrow 0$. Thus the continuum limit does not exists. This implies that Brown and Hiley's [25] extension of Bohmian mechanics to include the momentum representation (the momentum projector is the preferred projective measure) is not the continuum limit of Bell's modal dynamics. It is possible that a different choice for $J_{n m}(t)$ (and $T_{n m}(t)$ ) would allow their equations to be derived, but that is beyond the scope of this paper.

\section{B. The Velocity Operator Technique}

In the above section we have demonstrated that when using the Bell solution for the transition rates, modal dynamics for some continuous properties only reduces to a deterministic theory (apart from a random initial conditions) if the Hamiltonian is at most quadratic in the conjugate variable to the property. If the property is position then this deterministic limit is Bohmian mechanics and the trajectories are then found using Eq. (3.5), which requires calculation of the probability current density, $J_{k}\left(\left\{x_{j}\right\}, t\right)$. Here we present an alternative to this, a method to calculate $v_{k}\left(\left\{x_{j}\right\}, t\right)$ directly. We assume that

$$
v_{k}\left(\left\{x_{j}\right\}, t\right)=\frac{\operatorname{Re}\left[\left\langle\Psi(t) \mid\left\{x_{j}\right\}\right\rangle\left\langle\left\{x_{j}\right\}\left|\hat{v}_{k}(t)\right| \Psi(t)\right\rangle\right]}{\left\langle\Psi(t) \mid\left\{x_{j}\right\}\right\rangle\left\langle\left\{x_{j}\right\} \mid \Psi(t)\right\rangle},
$$

where $\hat{v}_{k}(t)$ is the $k^{\text {th }}$ component of the velocity operator. This operator is defined as

$$
\hat{v}_{k}(t)=-i\left[\hat{X}_{k}, \hat{H}_{\mathrm{uni}}(t)\right]
$$

To show that this does give the same trajectories as Bohmian mechanics, we note that the numerator of Eq. (3.37) should be $J_{k}\left(\left\{x_{j}\right\}, t\right)$ by definition. Now using the Hamiltonian defined in Eq. (3.6), the velocity operator is

$$
\hat{v}_{k}(t)=\hat{B}_{k}(t)+\hat{B}_{k}^{\dagger}(t)+2 \hat{C}_{k}(t) \hat{Y}_{k}+2 \hat{Y}_{k} \hat{C}_{k}^{\dagger}(t) .
$$

This results in the following velocity field

$$
\begin{aligned}
v_{k}\left(\left\{x_{j}\right\}, t\right)= & \frac{1}{\left\langle\tilde{\psi}\left(\left\{x_{j}\right\}, t\right) \mid \tilde{\psi}\left(\left\{x_{j}\right\}, t\right)\right\rangle} \operatorname{Re}\left[\langle \tilde { \psi } ( \{ x _ { j } \} , t ) | \left\{2 \operatorname{Re}\left[\hat{B}_{k}\left(\left\{x_{j}\right\}, t\right)\right]-4 i \operatorname{Re}\left[\hat{C}_{k}\left(\left\{x_{j}\right\}, t\right)\right] \partial_{x_{k}}\right.\right. \\
& \left.\left.-2 i \partial_{x_{k}}\left[\hat{C}_{k}^{\dagger}\left(\left\{x_{j}\right\}, t\right)\right]\right\}\left|\tilde{\psi}\left(\left\{x_{j}\right\}, t\right)\right\rangle\right] .
\end{aligned}
$$

Comparing this with Eq. (3.9) we see that the numerator is indeed $J_{k}\left(\left\{x_{j}\right\}, t\right)$. This completes our proof that our velocity method does generate the same trajectories as Bohmian mechanics for Hamiltonians of the form dis- played in Eq. (3.6). However, by extending this argument to higher orders it can shown that our velocity method does not agree with Bohmian mechanics. That is, this is another example of a method that only works for Hamil- 
tonians that do not contain terms of order $\hat{Y}_{k}^{3}$ and higher.

\section{SIMPLE EXAMPLE - HARMONIC OSCILLATOR}

\section{A. Husimi POM}

To illustrate modal dynamics for POMs, we investigate a simple model; a universe consisting of a one dimensional harmonic oscillator of frequency $\omega$. That is,

$$
\hat{H}_{\mathrm{uni}}(t)=\omega \hat{a}^{\dagger} \hat{a}
$$

where $\hat{a}^{\dagger}$ and $\hat{a}$ are the creation and annihilation operators of the harmonic oscillator respectively.

The preferred POM we consider is that of Ref. [22], which has POM elements (or effects) given by

$$
\hat{F}_{\alpha}=\frac{1}{\pi}|\alpha\rangle\langle\alpha| d^{2} \alpha
$$

where $\hat{a}|\alpha\rangle=\alpha|\alpha\rangle$. The preferred observable (one of the many) we associate with this POM is

$$
A=\left\{\left(\alpha, \frac{1}{\pi}|\alpha\rangle\langle\alpha| d^{2} \alpha\right)\right\}
$$

The continuous value $\alpha=x^{+}+i y^{-}$is a complex number representing a point is phase space $\left(x^{+}, y^{-}\right)$. In the orthodox theory this POM corresponds to a measure of both position and momentum with minimal additional uncertainty [18].

Before analyzing the modal dynamics that corresponds to this POM, we need to define a few operators that act in the enlarged Hilbert space $\mathcal{K}$. (The auxiliary Hilbert space $\mathcal{H}_{\text {aux }}$ is assumed to be a single harmonic oscillator). We define

$$
\begin{aligned}
& \hat{x}^{+}=\left[\hat{a}+\hat{a}^{\dagger}+\hat{b}+\hat{b}^{\dagger}\right] / 2, \\
& \hat{x}^{-}=\left[\hat{a}+\hat{a}^{\dagger}-\hat{b}-\hat{b}^{\dagger}\right] / 2, \\
& \hat{y}^{+}=\left[-i \hat{a}+i \hat{a}^{\dagger}-i \hat{b}+i \hat{b}^{\dagger}\right] / 2, \\
& \hat{y}^{-}=\left[-i \hat{a}+i \hat{a}^{\dagger}+i \hat{b}-i \hat{b}^{\dagger}\right] / 2,
\end{aligned}
$$

where $\hat{b}$ and $\hat{b}^{\dagger}$ are annihilation and creation operators which act in $\mathcal{H}_{\text {aux }}$. These four operators obey the commutator relations

$$
\begin{aligned}
& {\left[\hat{x}^{+}, \hat{y}^{+}\right]=\left[\hat{x}^{-}, \hat{y}^{-}\right]=i} \\
& {\left[\hat{x}^{+}, \hat{y}^{-}\right]=\left[\hat{x}^{-}, \hat{y}^{+}\right]=0}
\end{aligned}
$$

thus $\hat{x}^{+}$and $\hat{y}^{-}$have the same eigenstates, which we denote as $\left|x^{+}, y^{-}\right\rangle$. They are given by

$$
\left|x^{+}, y^{-}\right\rangle=\int \frac{d x^{\prime}}{\sqrt{2 \pi}}\left|\frac{x^{+}-x^{\prime}}{\sqrt{2}}\right\rangle_{\mathrm{aux}}\left|\frac{x^{+}+x^{\prime}}{\sqrt{2}}\right\rangle_{\mathrm{uni}} e^{i y^{-} x^{\prime}},
$$

where $\left|\left(x^{+}+x^{\prime}\right) / \sqrt{2}\right\rangle_{\text {uni }}$ is an $x$-state (an eigenstate of $\left.\hat{X}=\left(\hat{a}+\hat{a}^{\dagger}\right) / \sqrt{2}\right)$.

Because $\hat{x}^{+}$and $\hat{y}^{-}$can be well-defined simultaneously, we interpreted them as being suitable modal properties to represent simultaneously the position and momentum of the harmonic oscillator. This can be justified on the grounds that for $|\phi\rangle=|0\rangle$ (a vacuum state)

$$
\begin{aligned}
\left\langle\Phi(t)\left|\hat{x}^{+}\right| \Phi(t)\right\rangle & =\langle\Psi(t)|\hat{X}| \Psi(t)\rangle / \sqrt{2}, \\
\left\langle\Phi(t)\left|\hat{y}^{-}\right| \Phi(t)\right\rangle & =\langle\Psi(t)|\hat{Y}| \Psi(t)\rangle / \sqrt{2}, \\
\left\langle\Phi(t)\left|\hat{x}^{+} \hat{x}^{+}\right| \Phi(t)\right\rangle & =\left\langle\Psi(t)\left|\hat{X}^{2}\right| \Psi(t)\right\rangle / 2+1 / 4, \\
\left\langle\Phi(t)\left|\hat{y}^{-} \hat{y}^{-}\right| \Phi(t)\right\rangle & =\left\langle\Psi(t)\left|\hat{Y}^{2}\right| \Psi(t)\right\rangle / 2+1 / 4,
\end{aligned}
$$

where $|\Phi(t)\rangle=|\Psi(t)\rangle|0\rangle$ is the guiding wave for $\mathcal{K}$ and $|\Psi(t)\rangle$ is the solution of the Schrödinger equation. Thus these operators have essentially the same statistics as the position $(\hat{X})$ and momentum $\left(\hat{Y}=\left(-i \hat{a}+i \hat{a}^{\dagger}\right) / \sqrt{2}\right)$ operators in the classical limit (as the $1 / 4$ term becomes negligible).

In $\mathcal{K}$ we can rewrite $\hat{H}_{\text {uni }}$ as

$$
\begin{aligned}
\hat{H}_{\text {uni }} \otimes \hat{1}_{\text {aux }}= & \omega\left[\hat{x}^{+} \hat{x}^{+}+\hat{x}^{-} \hat{x}^{-}+\hat{y}^{+} \hat{y}^{+}+\hat{y}^{-} \hat{y}^{-}\right. \\
& \left.+2 \hat{x}^{+} \hat{x}^{-}+2 \hat{y}^{+} \hat{y}^{-}-2\right] / 4 .
\end{aligned}
$$

To define the modal dynamics in $\mathcal{K}$ we use Naimark theorem and a Naimark projector $|\phi\rangle=|0\rangle$ to extend the POM elements defined by Eq. (4.2) to the projector $\left|x^{+}, y^{-}\right\rangle\left\langle x^{+}, y^{-}\right| d x^{+} d y^{-}$. That is

$$
\frac{1}{\pi}|\alpha\rangle\langle\alpha| d^{2} \alpha=\left\langle 0 \mid x^{+}, y^{-}\right\rangle\left\langle x^{+}, y^{-} \mid 0\right\rangle d x^{+} d y^{-}
$$

(see appendix A). The observable $A$ becomes a property, which in matrix notation is given by

$$
\hat{A}=\iint\left(x^{+}+i y^{-}\right)\left|x^{+}, y^{-}\right\rangle\left\langle x^{+}, y^{-}\right| d x^{+} d y^{-}
$$

Since the preferred projective measure in $\mathcal{K}$, $\left\{\hat{\Pi}\left(x^{+}, y^{-}\right) d x^{+} d y^{-}=\left|x^{+}, y^{-}\right\rangle\left\langle x^{+}, y^{-}\right| d x^{+} d y^{-}\right\}$forms a complete orthogonal set and Eq. (4.15) contains no cubic or higher order terms involving $\hat{x}^{-}$or $\hat{y}^{+}$, the results of Sec. III B are applicable to this paper. That is a deterministic differential equation for the values $x^{+}(t)$ and $y^{-}(t)$ can be determined.

Using Eq. (3.38) with the Hamiltonian Eq. (4.15) gives the following two velocity operators

$$
\begin{aligned}
& \hat{v}_{+}(t)=\frac{\omega}{2} \hat{y}^{+}+\frac{\omega}{2} \hat{y}^{-}, \\
& \hat{v}_{-}(t)=-\frac{\omega}{2} \hat{x}^{+}-\frac{\omega}{2} \hat{x}^{-} .
\end{aligned}
$$

Substituting these into Eq. (3.37) (with $|\Psi(t)\rangle \rightarrow|\Phi(t)\rangle$ ) gives 


$$
\begin{aligned}
& v_{+}\left(x^{+}, y^{-}, t\right)=\frac{\omega \operatorname{Re}\left[-i\left\langle\Phi(t) \mid x^{+}, y^{-}\right\rangle \partial_{x^{+}}\left\langle x^{+}, y^{-} \mid \Phi(t)\right\rangle\right]}{2\left\langle\Phi(t) \mid x^{+}, y^{-}\right\rangle\left\langle x^{+}, y^{-} \mid \Phi(t)\right\rangle}+\frac{\omega}{2} y^{-}, \\
& v_{-}\left(x^{+}, y^{-}, t\right)=-\frac{\omega \operatorname{Re}\left[i\left\langle\Phi(t) \mid x^{+}, y^{-}\right\rangle \partial_{y^{-}}\left\langle x^{+}, y^{-} \mid \Phi(t)\right\rangle\right]}{2\left\langle\Phi(t) \mid x^{+}, y^{-}\right\rangle\left\langle x^{+}, y^{-} \mid \Phi(t)\right\rangle}-\frac{\omega}{2} x^{+} .
\end{aligned}
$$

Thus the differential equations are

$$
\begin{aligned}
& d_{t} x^{+}(t)=\left.\frac{\omega \operatorname{Re}\left[-i\left\langle\Phi(t) \mid x^{+}, y^{-}\right\rangle \partial_{x^{+}}\left\langle x^{+}, y^{-} \mid \Phi(t)\right\rangle\right]}{2\left\langle\Phi(t) \mid x^{+}, y^{-}\right\rangle\left\langle x^{+}, y^{-} \mid \Phi(t)\right\rangle}\right|_{x^{+}=x^{+}(t), y^{-}=y^{-}(t)}+\frac{\omega}{2} y^{-}(t), \\
& d_{t} y^{-}(t)=-\left.\frac{\omega \operatorname{Re}\left[i\left\langle\Phi(t) \mid x^{+}, y^{-}\right\rangle \partial_{y^{-}}\left\langle x^{+}, y^{-} \mid \Phi(t)\right\rangle\right]}{2\left\langle\Phi(t) \mid x^{+}, y^{-}\right\rangle\left\langle x^{+}, y^{-} \mid \Phi(t)\right\rangle}\right|_{x^{+}=x^{+}(t), y^{-}=y^{-}(t)}-\frac{\omega}{2} x^{+}(t) .
\end{aligned}
$$

Using the fact that (see appendix A)

$$
\left\langle x^{+}, y^{-} \mid \Phi(t)\right\rangle=\frac{\exp \left[-\left(x^{+^{2}}+y^{-2}\right) / 2\right]}{\sqrt{\pi}} \sum_{m} \frac{\left(x^{+}-i y^{-}\right)^{m}}{\sqrt{m !}}\langle m \mid \Psi(t)\rangle
$$

the partial derivatives can be written as

$$
\begin{aligned}
\partial_{x^{+}}\left\langle x^{+}, y^{-} \mid \Phi(t)\right\rangle & =\frac{\exp \left[-\left(x^{+^{2}}+y^{-2}\right) / 2\right]}{\sqrt{\pi}} \sum_{m} m \frac{\left(x^{+}-i y^{-}\right)^{m-1}}{\sqrt{m !}}\langle m \mid \Psi(t)\rangle-x^{+}\left\langle x^{+}, y^{-} \mid \Phi(t)\right\rangle, \\
\partial_{y^{-}}\left\langle x^{+}, y^{-} \mid \Phi(t)\right\rangle & =-i \frac{\exp \left[-\left(x^{+^{2}}+y^{-2}\right) / 2\right]}{\sqrt{\pi}} \sum_{m} m \frac{\left(x^{+}-i y^{-}\right)^{m-1}}{\sqrt{m !}}\langle m \mid \Psi(t)\rangle-y^{-}\left\langle x^{+}, y^{-} \mid \Phi(t)\right\rangle .
\end{aligned}
$$

which allows us to write

$$
\begin{aligned}
& d_{t} x^{+}(t)=+\frac{\omega}{2} y^{-}(t)+\frac{\omega}{2} \operatorname{Im}\left[\chi_{\psi}\left(x^{+}(t), y^{-}(t)\right)\right], \\
& d_{t} y^{-}(t)=-\frac{\omega}{2} x^{+}(t)-\frac{\omega}{2} \operatorname{Re}\left[\chi_{\psi}\left(x^{+}(t), y^{-}(t)\right)\right],
\end{aligned}
$$

where

$\chi_{\psi}\left(x^{+}(t), y^{-}(t)\right)=\frac{\sum_{m} m\left(x^{+}-i y^{-}\right)^{m-1}\langle m \mid \Psi(t)\rangle / \sqrt{m !}}{\sum_{m}\left(x^{+}-i y^{-}\right)^{m}\langle m \mid \Psi(t)\rangle / \sqrt{m !}}$.

Thus the differential equation for $\alpha(t)$ is

$$
d_{t} \alpha(t)=-\frac{i \omega}{2} \alpha(t)-\frac{i \omega}{2} \chi_{\psi}\left(x^{+}(t), y^{-}(t)\right)
$$

\section{When $|\Psi(t)\rangle$ is a number state}

Lets first of all consider a number state $|n\rangle$, as the initial condition for $|\Psi(0)\rangle$. Then by the Schrödinger equation

$$
d_{t}|\Psi(t)\rangle=-i \omega \hat{a}^{\dagger} \hat{a}|\Psi(t)\rangle
$$

$|\Psi(t)\rangle=e^{-i \omega n t}|n\rangle$. Substituting this into Eq. (4.29), gives

$$
\begin{aligned}
\chi_{\psi}\left(x^{+}(t), y^{-}(t)\right)= & \frac{n\left[x^{+}(t)-i y^{-}(t)\right]^{n-1}}{\left[x^{+}(t)-i y^{-}(t)\right]^{n}}=\frac{n}{\alpha^{*}(t)} \\
& =\frac{n \alpha(t)}{|\alpha(t)|^{2}} .
\end{aligned}
$$

Thus

$$
d_{t} \alpha(t)=-\frac{i \omega}{2}\left(1+\frac{n}{|\alpha(t)|^{2}}\right) \alpha(t) .
$$

This has the solution

$$
\alpha(t)=\alpha(0) e^{-i \omega^{\prime} t}
$$

where $\omega^{\prime}=\omega\left(1+n /|\alpha(0)|^{2}\right) / 2$. This solution and all subsequent solutions are discussed in Sec. IV C.

\section{When $|\Psi(t)\rangle$ is a coherent state}

If we assume that initially the system in is a coherent state $|\Psi(0)\rangle=|\beta\rangle$, then by Eq. (4.31),

$$
|\Psi(t)\rangle=\exp \left(|\beta|^{2} / 2\right) \sum_{n} \frac{\beta^{n} e^{-i \omega n t}}{\sqrt{n !}}|n\rangle .
$$


Substituting this into Eq. (4.29), gives

$$
\begin{aligned}
\chi_{\psi}\left(x^{+}(t), y^{-}(t)\right) & =\beta e^{-i \omega t} \frac{\sum_{m} m\left(\alpha^{*} \beta e^{-i \omega t}\right)^{m-1} / m !}{\sum_{m}\left(\alpha^{*} \beta e^{-i \omega t}\right)^{m} / m !} \\
& =\beta e^{-i \omega t} .
\end{aligned}
$$

Thus

$$
d_{t} \alpha(t)=-\frac{i \omega}{2} \alpha(t)-\frac{i \omega}{2} \beta e^{-i \omega t} .
$$

This has the solution

$$
\alpha(t)=[\alpha(0)-\beta] e^{-i \omega t / 2}+\beta e^{-i \omega t} .
$$

\section{B. Bohmian Mechanics, The Position Projector}

In this section we consider the modal dynamics which describe a decomposition into position eigenstates. That is the preferred projective measure is $\{\hat{\pi}(x) d x=$ $|x\rangle\langle x| d x\}$. Since this is already a projector, there is no need to enlarge the universe, and as shown above (and in Ref. [4] and Ref. [5]) the modal dynamics for this case is just Bohmian mechanics.

In terms of $\hat{X}$ and its conjugate operator $\hat{Y}$, the Hamiltonian for the universe, Eq. (4.1), becomes $\hat{H}_{\text {uni }}(t)$

$$
\hat{H}_{\text {uni }}(t)=\omega\left(\hat{X}^{2}+\hat{Y}^{2}-1\right) / 2 .
$$

Using our velocity operator technique it can easily be shown that the velocity field is

$$
v(x, t)=\frac{\omega \operatorname{Re}\left[-i\langle\Psi(t) \mid x\rangle \partial_{x}\langle x \mid \Psi(t)\rangle\right]}{\langle\Psi(t) \mid x\rangle\langle x \mid \Psi(t)\rangle},
$$

as $\hat{v}=\omega \hat{Y}$. Using Eq. (3.5) this gives

$$
d_{t} x(t)=\left.\frac{\omega \operatorname{Im}\left[\langle\Psi(t) \mid x\rangle \partial_{x}\langle x \mid \Psi(t)\rangle\right]}{\langle\Psi(t) \mid x\rangle\langle x \mid \Psi(t)\rangle}\right|_{x=x(t)} .
$$

Since $\hat{Y}$ does not commute with $\hat{X}$, we can not give both $\hat{X}$ and $\hat{Y}$ definite status (to give $\hat{Y}$ property status we would have to chose a momentum projective measure as the preferred projective measure). However, as in Bohmian mechanics, we can define a momentum field, $y(x, t)$, by

$$
\begin{aligned}
y(x, t) & =\frac{\operatorname{Re}[\langle\Psi(t) \mid x\rangle\langle x|\hat{y}| \Psi(t)\rangle]}{\langle\Psi(t) \mid x\rangle\langle x \mid \Psi(t)\rangle} \\
& =\frac{\operatorname{Im}\left[\langle\Psi(t) \mid x\rangle \partial_{x}\langle x \mid \Psi(t)\rangle\right]}{\langle\Psi(t) \mid x\rangle\langle x \mid \Psi(t)\rangle} .
\end{aligned}
$$

One interprets this momentum field as follows: if the system has the position $x(t)$ then its momentum is $\left.y(x)\right|_{x=x(t)}$. With this and position $x(t)$ we can define a phase point $\left(x(t),\left.y(x)\right|_{x=x(t)}\right)$, which in complex notation is written as

$$
\alpha(t)=\frac{x(t)+\left.i y(x)\right|_{x=x(t)}}{\sqrt{2}} .
$$

The factor $1 / \sqrt{2}$ is to scale this to agree with the preceding section.

\section{When $|\Psi(t)\rangle$ is a number state}

As before when we assume a number state initial condition the guiding wave at time $t$ is $|\Psi(t)\rangle=e^{-i \omega n t}|n\rangle$. Substituting this into Eq. (4.41) and using

$$
|x\rangle=\frac{1}{\pi^{1 / 4}} \exp \left(-x^{2} / 2\right) \sum_{n} \frac{H_{n}(x)}{\sqrt{2^{n} n !}}|n\rangle
$$

where $H_{n}(x)$ is a $n^{\text {th }}$ order Hermite polynomial,

$$
\begin{aligned}
d_{t} x(t) & =\left.\frac{\omega \operatorname{Im}\left\{\partial_{x}\left[\exp \left(-x^{2} / 2\right) H_{n}(x)\right] / \sqrt{2^{n} n !}\right\}}{\exp \left(-x^{2} / 2\right) H_{n}(x) / \sqrt{2^{n} n !}}\right|_{x=x(t)} \\
& =0 .
\end{aligned}
$$

Using a similar argument and Eq. (4.42) we get $y(x, t)_{x=x(t)}=0$. That is, once the initial $x(0)$ is picked from the quantum mechanical distribution is stays there for all time. In terms of the complex notation $\alpha(t)$ we get

$$
\alpha(t)=x(0) / \sqrt{2}
$$

\section{When $|\Psi(t)\rangle$ is a coherent state}

If we assume that initially the system in is a coherent state $|\Psi(0)\rangle=|\beta\rangle$, then Eq. (4.35) in the position representation is

$$
\begin{aligned}
|\Psi(t)\rangle= & \frac{\exp \left(|\beta|^{2} / 2\right)}{\pi^{1 / 4}} \int d x^{\prime} \exp \left(\sqrt{2} \beta e^{-i \omega t} x\right. \\
& \left.-\beta^{2} e^{-i 2 \omega t} / 2\right) \exp \left(-x^{\prime 2} / 2\right)\left|x^{\prime}\right\rangle .
\end{aligned}
$$

Substituting this into Eq. (4.41) gives

$$
\begin{aligned}
d_{t} x(t) & =\left.\frac{\omega \operatorname{Im}\left\{\partial_{x}\left[\exp \left(-x^{\prime 2} / 2\right) \exp \left(\sqrt{2} \beta e^{-i \omega t} x\right)\right]\right\}}{\exp \left(-x^{\prime 2} / 2\right) \exp \left(\sqrt{2} \beta e^{-i \omega t} x\right)}\right|_{x=x(t)} \\
& =\omega \sqrt{2} \operatorname{Im}\left[\beta e^{-i \omega t}\right],
\end{aligned}
$$

and using Eq. (4.42) we get $y(x, t)_{x=x(t)}=\sqrt{2} \operatorname{Im}\left[\beta e^{-i \omega t}\right]$. Taking the derivative of this gives

$$
d_{t} y(x, t)_{x=x(t)}=-\omega \sqrt{2} \operatorname{Re}\left[\beta e^{-i \omega t}\right] .
$$

Thus

$$
d_{t} \alpha(t)=\omega\left\{\operatorname{Im}\left[\beta e^{-i \omega t}\right]-i \operatorname{Re}\left[\beta e^{-i \omega t}\right]\right\}=-i \omega \beta e^{-i \omega t} .
$$

This has the solution

$$
\alpha(t)=\beta e^{-i \omega t}+(\alpha(0)-\beta) .
$$

\section{Classical Limit}

The classical harmonic oscillator has the well known solution, this being

$$
\alpha(t)=\alpha(0) e^{-i \omega t}
$$


In Sec. IV A (modal dynamics when the preferred POM is the Husimi POM) we saw that in the enlarged Hilbert space, we can define $\alpha(t)=x^{+}(t)+i y^{-}(t)$. Now consider the classical limit. First consider the case of the number state Eq. (4.34). From the probability formula (2.22) it can be shown that $|\alpha(t)|^{2} \approx n$ with high probability. This means that as $n \rightarrow \infty, \omega^{\prime} \rightarrow \omega$, reproducing the classical dynamics. When considering the case with an initial coherent state we can similarly argue that $\beta \approx \alpha(0)$ with high probability. Then in the limit $|\beta| \rightarrow \infty$, the difference between the classical formula and Eq. (4.38) is negligible.

By contrast, in the position case (Bohmian mechanics), for the first case (number state), $\alpha(t)=x(0)$ for all $n$, the dynamics is completely non-classical. However, it can be argued that when we consider the second case with an initial coherent state, again the difference between the classical formula and Eq. (4.51) is negligible. The fact that in both Bohmian mechanics and the modal interpretation of Sec. IV A have a good classical correspondence for coherent state is not surprising, as the coherent state is a classical-like state. What is surprising is that it is possible to obtain classical modal dynamics even for a non-classical state, by using POMs.

\section{DISCUSSION}

In this paper we have extended the modal dynamics of Refs. $[3,4,5,7,8,9]$ to include the possibility of having a preferred POM. To do this we enlarged the Hilbert space from $\mathcal{H}_{\text {uni }}$ to $\mathcal{K}=\mathcal{H}_{\text {uni }} \otimes \mathcal{H}_{\text {aux }}$. Once in this enlarged Hilbert space we used Naimark's theorem [18, 24] to define a preferred projective measure which is equivalent to the preferred POM. That is the statistics of an observable describe by $Z=\left\{\left(z_{n}, \hat{F}_{n}(t)\right)\right\}$, (where $\hat{F}_{n}(t)$ is a POM element) is equivalent to the statistic of the property $Z=\left\{\left(z_{n}, \hat{\Pi}_{n}(t)\right)\right\}$. Here $\hat{\Pi}(t)$ is a projector in $\mathcal{K}$ and defines the property state $\left|\Phi_{n}(t)\right\rangle$. This state represents the actual state of the universe and determines the present value, $z_{n}$, of the property $\hat{Z}$ from the set of possible values. The evolution (jumping between $z_{n}$ ) is determined by the stochastic evolution of $\left|\Phi_{n}(t)\right\rangle$, which in-turn depends on the guiding wave $|\Phi(t)\rangle=|\phi\rangle|\Psi(t)\rangle$. $|\Psi(t)\rangle$ is the standard quantum state found from the Schrödinger equation and $|\phi\rangle$ is a state defined in the auxiliary Hilbert space.

To illustrate modal dynamics for POMs we considered a simple example: a universe consisting of a single Harmonic oscillator. To illustrate our new dynamics, we looked at the Husimi POM and compared the dynamics obtained to that which is obtained with the position projective measure (Bohmian mechanics). Since the first case corresponds to a POM we have to use our enlarged Hilbert space modal dynamics to develop the stochastic evolution equation for the value of the property (or equivalently the property state). For the Husimi POM we denoted this value by $\alpha(t)$. We find that by choosing a Naimark projector $|\phi\rangle=|0\rangle$, this property defines a point in phase space $\left(\alpha(t)=x^{+}+i y^{-}\right)$that is effectively the position and momentum of the system. Investigating two different initial conditions for $|\Psi(0)\rangle$, namely a number state and coherent state, we find that the differential equation for $\alpha(t)$ for both cases has a classical limit which agrees with classical theories. When comparing to the modal dynamics for the position projective measure we find that when the initial state is the number state the dynamics are highly non-classical. Only for an initial coherent state (a classical-like state) can a classical limit can be obtained.

In conclusion by extending modal dynamics to include POMs allows the ability to include overcomplete decomposition, like the Husimi POM $[22,23]$ and informationally complete POMs $[20,21]$. This may provide an answer to questions involving the quantum-classical limit. An interesting question for future work is whether the extension of the Hilbert space is only a mathematical tool or whether there is some physical significance behind enlarging the Hilbert space. Apart from this fundamental question we intend to use this theory to explain diffusive non-Markovian stochastic schrodinger equations (SSEs) [26]. In a recent paper we have shown that under the orthodox interpretation non-Markovian SSEs represent nothing more then a stencil which determines the state of a system at a particular time $t$, given that a measurement on the environment at that time yielded result $z$ [27]. That is, non-Markovian SSEs can not be interpreted as evolution equations for the state of the system conditioned on the outcomes of some continuous measurement of the environment. We believe that under the modal theory it can be shown that non-Markovian SSEs are evolution equations for the system part of the property state when the environment property $Z$ is given definite status. In [28] we have shown that when the preferred measure is position, non-Markovian SSEs do have this interpretation. To interpret other non-Markovian SSEs $[26,27]$, it is necessary to consider non-orthogonal decompositions.

APPENDIX A: PROOF OF EQ. (4.16). 
To show that $|\alpha\rangle\langle\alpha| / \pi=\left\langle 0 \mid x^{+}, y^{-}\right\rangle\left\langle x^{+}, y^{-} \mid 0\right\rangle$ we consider just $\left\langle 0 \mid x^{+}, y^{-}\right\rangle=|\alpha\rangle / \sqrt{\pi}$. Using Eq. (4.10), and the standard definition of a $x$-state Eq. (4.44), $\left\langle 0 \mid x^{+}, y^{-}\right\rangle$becomes

$$
\left\langle 0 \mid x^{+}, y^{-}\right\rangle=\int \frac{d x^{\prime}}{\sqrt{2 \pi}} \frac{1}{\pi^{1 / 4}} \exp \left[\frac{-\left(x^{+}-x^{\prime}\right)^{2}}{4}\right]\left|\frac{x^{+}+x^{\prime}}{\sqrt{2}}\right\rangle_{\text {uni }} e^{i y^{-} x^{\prime}} .
$$

Defining $X=\left(x^{+}+x^{\prime}\right) / \sqrt{2}$ allows us to rewrite this as

$$
\left\langle 0 \mid x^{+}, y^{-}\right\rangle=\int \frac{d X}{\sqrt{\pi}} \frac{1}{\pi^{1 / 4}} \exp \left[\frac{-\left(2 x^{+}-\sqrt{2} X\right)^{2}}{4}\right]|X\rangle_{\text {uni }} \sqrt{2} e^{i y^{-}\left(\sqrt{2} X-x^{+}\right)},
$$

which with definition Eq. (4.44) can be expanded to

$$
\begin{aligned}
\left\langle 0 \mid x^{+}, y^{-}\right\rangle & =\sum_{n} \int \frac{d X}{\pi} \exp \left(-x^{+} x^{+}+\sqrt{2} X x^{+}-X^{2}+i y^{-} \sqrt{2} X-i y^{-} x^{+}\right) \frac{H_{n}(X)}{\sqrt{2^{n} n !}}|n\rangle_{\text {uni }} \\
& =\exp \left(-|\alpha|^{2} / 2\right) \sum_{n} \int \frac{d X}{\pi} \exp \left(-X^{2}\right) \exp \left(2 \alpha X / \sqrt{2}-\alpha^{2} / 2\right) \frac{H_{n}(X)}{\sqrt{2^{n} n !}}|n\rangle_{\text {uni }}
\end{aligned}
$$

where $\alpha=x^{+}+i y^{-}$. Then using $\sum_{m} t^{m} H_{m}(x) / m !=\exp \left(2 t x-t^{2}\right)$ this can be written as

$$
\left\langle 0 \mid x^{+}, y^{-}\right\rangle=\frac{\exp \left(-|\alpha|^{2} / 2\right)}{\pi} \sum_{n, m} \frac{\alpha^{m}}{\sqrt{2^{m}} m ! \sqrt{2^{n} n !}}|n\rangle_{\text {uni }} \int d X \exp \left(-X^{2}\right) H_{m}(X) H_{n}(X)
$$

and since $\int d X \exp \left(-X^{2}\right) H_{m}(X) H_{n}(X)=2^{n} n ! \sqrt{\pi} \delta_{n m}$ this becomes

$$
\left\langle 0 \mid x^{+}, y^{-}\right\rangle=\frac{\exp \left(-|\alpha|^{2} / 2\right)}{\sqrt{\pi}} \sum_{n} \frac{\alpha^{n}}{\sqrt{n !}}|n\rangle_{\text {uni }}=\frac{1}{\sqrt{\pi}}|\alpha\rangle_{\text {uni }}
$$

Thus $|\alpha\rangle\langle\alpha| / \pi=\left\langle 0 \mid x^{+}, y^{-}\right\rangle\left\langle x^{+}, y^{-} \mid 0\right\rangle$.

\section{ACKNOWLEDGMENTS}

We would like to thank R. Spekkens for useful discussions. We would also like to thank an unnamed referee who provided very useful comments. We believe these comments have improved the readability of the paper. This work was supported by the ARC.
[1] Heisenberg W.: The Physical Principles of Quantum Mechanics. The University of Chicago Press, Chicago, 1930.

[2] von Neumann J.: Mathematical Foundations of Quantum Mechanics. Springer, Berlin, 1932; English translation, Princeton University Press, Princeton, 1955.

[3] Bell J. S.: 1984, "Beables for quantum field theory," CERN-TH.4035/84. Reprinted in John S. Bell on the Foundations of Quantum Mechanics, edited by M. Bell, K. Gottfried, and M. Veltman, World Scientific, Singapore, 2001.

[4] Sudbery A.: 1987, "Objective interpretations of quantum mechanics and the possibility of a deterministic limit," $J$. Phys. A 20, 1743.

[5] Vink J.C.: 1993, "Quantum mechanics in terms of discrete beables," Phys. Rev. A 48, 1808.

[6] Bub J.: Interpretating the Quantum World. Cambridge University Press, Cambridge, 1997.

[7] Sudbery A.: 2002, "Diese Verdammte Quantenspringerei," Stud. Hist. Phil. Mod. Phys. 33, 387.
[8] Dickson M.: 1997, "On the plurality of dynamics: transition probabilities and modal interpretations," p.160. In Healey R. A., and Hellman G., Quantum Measurements: Beyond Paradox. University of Minnesota Press, Minneapolis, 1997.

[9] Bacciagaluppi G. and M. Dickson: 1999, "Dynamics for modal interpretations," Found. Phys. 29, 1165.

[10] Vermass P. E., and D. Dieks: 1995, "The modal interpretation of quantum mechanics and its generalization to density operators," Found. Phys. 25, 145.

[11] Dieks D.: 1995, "Physical motivation of the modal interpretations of quantum mechanics," Phys. lett. A 197, 367.

[12] Dieks D.: "Preferred factorizations and consistent property attribution," p.144. In R. A. Healey and G. Hellman, Quantum Measurements: Beyond Paradox. University of Minnesota Press, Minneapolis, 1997.

[13] Healy R., The Philosophy of Quantum Mechanics: An Interactive Interpretation. Cambridge University Press, 
Cambridge, 1989.

[14] Spekkens R. W. and J. E. Sipe: 2001a, "Non-orthogonal core projectors for modal interpretations of quantum mechanics," Found. Phys. 31, 1403.

[15] Spekkens R. W., and J. E. Sipe: 2001b, "A modal interpretations of quantum mechanics based on a principle of entropy minimization," Found. Phys. 31, 1431.

[16] Bohm D.: 1952, "A suggested interpretation of quantum theory in terms of hidden variables," Phys. Rev. 85, 166; ibid. 180.

[17] Bacciagaluppi G.: 1995, "Kochen-Specker theorem in the modal interpretation of quantum mechanics," Int. J. Theor. Phys. 34, 1205.

[18] Helstrom C. W.: Quantum Detection and Estimation Theory. Academic Press, New York, 1976.

[19] K. Kraus: States, Effects, and Operations: Fundamental Notions in Quantum Theroy. Springer, Berlin, 1983.

[20] Prugovečki E.: 1977, Int. J. Theo. Phys. 16, 321.

[21] Caves C. M., C. A. Fuchs, R. Schack: 2002, "Unknown quantum states: The quantum de Finetti representation," J. Math. Phys. 43, 4537.

[22] Husimi K.: 1940, "Some formal properties of the density
Matrix," Proc. Phys. Math. Soc. Jpn. 22, 264

[23] Walls D. F., and G. J. Milburn: Quantum Optics. Springer, Berlin, 1994.

[24] Busch P., M. Grabowski and P.J. Lahti: Operational Quantum Physics. Springer, New York, 1995.

[25] Brown M. R., and B.J. Hiley: 2000, "Schrodinger revisited: an algebraic approach," quant-ph/0005026.

[26] Diósi L., N. Gisin, and W.T. Strunz: 1998, "NonMarkovian quantum state diffusion," Phys. Rev. A 58, 1699.

[27] Gambetta J., and H. M. Wiseman: 2002, "NonMarkovian Stochastic Schrodinger equations: Generalization to real-valued noise using quantum measurement theory," Phys. Rev. A 66, 012108.

[28] Gambetta J. and H. M. Wiseman: 2003, "A nonMarkovian stochastic Schrodinger equation developed from a hidden variable interpretation," pp 313-324. In Proceedings of SPIE 5111 Fluctuations and Noise in Photonics and Quantum Optics, edited by D. Abbott, J. H. Shapiro, and Y. Yamamoto, SPIE, Bellingham, 2003. 